\title{
The Effect of 8-Week Resistance Training and Leucine Supplementation on Protein Synthesis Among Elderly Men With Sarcopenia
}

\author{
Zahra Sadat Pormozafari", Mohsen Aminaie ${ }^{2}$, Rohollah Nikooie ${ }^{2}$ \\ ${ }^{1}$ Faculty of Sport Sciences, University of Shahid Bahonar Kerman, Kerman, Iran \\ ${ }^{2}$ Department of Exercise Physiology, Faculty of Sport Sciences, University of Shahid Bahonar Kerman, Kerman, Iran
}

\section{*Correspondence to}

Zahra Sadat Pormozafari, MSc; Department of Exercise Physiology, Faculty of Sport Sciences, University of Shahid Bahonar Kerman, Kerman, Iran.

Tel: +0989137589813

Email: Zahra.pormozafari@gmail.com

Received December 18, 2017 Accepted March 17, 2018 Published online June 30, 2018

\begin{abstract}
Introduction: The aim of the study was to determine the effect of eight-week resistance training and supplementation of leucine on protein synthesis in elderly men suffering from sarcopenia. Methods: A total of 40 elderly men residing in elderly nursing center were randomly selected and divided into 4 groups to be evaluated: Resistance training and leucine supplement (RTLS=10), resistance training $(R T=10)$, leucine supplement $(L S=10)$, and Control $(C=10)$. Resistance training was performed with intensity training of $35 \%-70 \%$ of one repetition maximum (1RM) for 20-45 minutes during an 8-week interval. The supplemented group consumed $3 \mathrm{~g}$ of leucine amino acid in every meal. The serum insulin concentration, urine nitrogen, muscle mass, and muscle strength were measured 1 day prior to the implementation of the protocol and 72 hours following the last training session. Analysis of covariance (ANCOVA) was analyzed within and between group changes. Bonferroni post hoc test was used to determine the difference between each of the 2 groups.

Results: The results showed that urine nitrogen and muscle mass levels increased significantly after 8 weeks of RT compared to $\mathrm{C}$ group $(P<0.05)$. Serum insulin concentration increased significantly in RT group compared to $\mathrm{C}$ group $(P<0.05)$. In addition, there was a significant increase in muscle strength in RT and $\mathrm{S}$ groups compared to $C$ group $(P<0.05)$.

Conclusion: The results indicated that muscle protein synthesis increased after resistance training in skeletal muscle. This implies that the rate of sarcopenia reduced as a result of resistance training. Likewise,, resistance training along with consumption of leucine supplement were effective in improving muscle strength.

Keywords: Resistance exercise, Leucine, Protein synthesis, Older men, Sarcopenia
\end{abstract}

Please cite this article as follows: Pormozafari ZS, Aminaie M, Nikooie R. The effect of 8-week resistance training and leucine supplementation on protein synthesis among elderly men with sarcopenia. Int J Basic Sci Med. 2018;3(2):83 88. doi:10.15171/ ijbms.2018.15.

\section{Introduction}

Muscle atrophy (loss of mass, strength, and endurance) during aging process is referred to as sarcopenia. ${ }^{1}$ Muscle mass is lost about $0.1 \%-0.5 \%$ annually. This starts at the third decade of life with a steep acceleration after the age of $65 .^{2}$ Among the possible causes of sarcopenia are malnutrition, poor quality of life, low physical activity, and physiological factors such as changes in insulin secretion, hormonal contractions, chronic inflammation, changes in endocrine function, etc. ${ }^{3,4}$ The age related muscular atrophy is primarily due to decreased protein content. ${ }^{1}$ This decrease is significant, remarkably as Balagopal et al found that muscle protein synthesis will be reduced about $30 \%$ with aging. ${ }^{5}$

To maintain homeostasis and function, cells must be continuously produced and decomposed into proteins. As the aging increases, the key factors of protein translation decrease, resulting in a decrease in the protein synthesis rate. ${ }^{6}$ Although different factors stimulate protein synthesis, a main share can be attributed to the amount of amino acids in a meal. ${ }^{7}$ Essential amino acids, especially leucine, play an important role in improving protein synthesis. ${ }^{8}$ Leucine regulates protein synthesis through insulin-dependent and independent mechanisms. ${ }^{3}$ The activation of mechanistic target of rapamycin (mTOR) leads to phosphorylation of

(c) 2018 The Author(s); Published by Zabol University of Medical Sciences. This is an open-access article distributed under the terms of the Creative Commons Attribution License (http://creativecommons.org/licenses/by/4.0), which permits unrestricted use, distribution, and reproduction in any medium, provided the original work is properly cited. 
4E-binding protein 1 (4E-Bp1) and S6 kinase beta-1 (S6K1) proteins, which are located in the pathway of protein synthesis. ${ }^{9}$ These 2 proteins activate the ribosomal protein S6 (rs6), eukaryotic translation initiation factor $4 \mathrm{f}$ (elf4f), and eukaryotic elongation factor 2 (eEf2), which initiate translation from the mRNA, ${ }^{9}$ and ultimately facilitate skeletal muscle hypertrophy. ${ }^{10}$ Leucine also stimulates insulin secretion. Insulin plays a main role in protein synthesis. ${ }^{3}$ Insulin has an anabolic effect on protein metabolism, which activates the protein synthesis reaction and prevents the breakdown, while increasing the absorption of amino acids by muscle cells. ${ }^{11}$

Physical inactivity is another important factor in reduction of muscle mass (sarcopenia). ${ }^{12}$ Resistance training has been shown to improve the translation process and increase the synthesis of contractile and structural proteins, the number of cell proliferation markers and their number, the number of nuclei of each muscle, as well as the differentiation of myoblasts and their fusion. ${ }^{13}$ Resistance training through stimulating signaling mTOR, which is a key component in regulating protein synthesis in skeletal muscle, leads to the stimulation of protein synthesis. ${ }^{14}$ A study carried out on old men with no sign of sarcopenia disease showed that both leucine and resistance training have a synergy effect and contribute to the development of protein synthesis in muscle and hypertrophy. ${ }^{15}$ Moreover, Drummond et al demonstrated that the consumption of $20 \mathrm{~g}$ of leucine amino acid after a resistance training session increases the stimulation of muscle protein synthesis compared to control group after exercising resistance training among healthy old men. In a study by Waters et al, it was revealed that the consumption of $15 \mathrm{~g}$ of leucine supplementation one hour after resistance training with a 70\% 1RM intensity of exercise increased protein synthesis in healthy elders. ${ }^{16}$ Willoughby et al indicated that resistance training with $85 \%-90 \% 1 \mathrm{RM}$ intensity consuming $20 \mathrm{~g}$ of leucine supplementation would regulate muscle protein synthesis and improve muscle performance in healthy elderly. ${ }^{17}$

The effects of simultaneous use of leucine supplement and exercise on protein synthesis among sarcopenia were the main gaps of this study not being observed in previous studies. Many researches have been conducted on healthy elderly men. The aim of this study is to determine the effect of training and consuming Lucien supplement on old men suffering from sarcopenia.

\footnotetext{
Methods

Subjects

A total of 40 elderly men (in the age range of 60-65 years) suffering from sarcopenia and residing in nursing houses were selected as the subjects of the study. Sarcopenia in the elderly is identified on the basis of hand muscle strength (fingers and forearm flexor) less than $26-30 \mathrm{~kg}$, the ability to walk $4 \mathrm{~m}$ in less than $0.8 \mathrm{~m} / \mathrm{s}$, and muscle mass index among young population with an average less than 2
}

standard deviations. ${ }^{18}$ After filling out the consent form and verifying the health status and exercise history, the subjects were grouped based on muscle hand strength less than $26-30 \mathrm{~kg}$, and were randomly divided into 4 equal groups: Resistance training and leucine supplemental (RTLS), resistance training (RT), leucine supplementation (LS), and control (C).

Exclusion criteria included a history of chronic diseases (heart failure, chronic obstructive lung, kidney failure, cancer, diabetes, anemia, and thyroid disease), drug or supplementation (such as corticosteroids and anabolic hormones), inability to exercise, and a regular exercise history ( 3 times a week, more than 30 minutes).

\section{Resistance Exercise Training}

The resistance exercise protocol was performed 3 sessions per week (for 8 weeks). At the beginning and the end of each session, the subjects were warmed up for 10 minutes and cooled down for 5 minutes, respectively. The intensity of resistance training started with $35 \%$ of 1 repetition maximum (1RM) for 20 minutes and the intensity and duration of the exercise were increased based on the principle of overload every week, reaching to $70 \%$ of $1 \mathrm{RM}$ for 45 minutes in the final week. Resistance training program consisted of 2 sets, 8 - 12 repetitions, and 2 minutes rest between each set. ${ }^{19}$

\section{Supplementation}

LS subjects received 3 g leucine supplement (TITRACHEM, 6105360, iRan) per day. Control (C) subjects consumed placebo with $200 \mathrm{~mL}$ of water per 3 days in every week. The RTLS subjects consumed $3 \mathrm{~g}$ of leucine supplement 1 hour before and 3 hours after exercise 3 days per week. ${ }^{14}$

\section{Protein Synthesis Analysis}

In the present study, protein synthesis was indirectly investigated based on changes in urine nitrogen and muscle mass. Muscle mass was measured by 4 points skinfold technic (using Meikosha model calipers (with an instrument accuracy of $0.2 \mathrm{~mm}$ ). The fasting blood insulin and urine nitrogen levels were determined at 7-8 in the morning. The urine nitrogen was analyzed and recorded using Biochemistry Analyzer (Autlab, Ames, Rome, Italy). The insulin was analyzed using Liason, DiaSorin kit and the sensitivity of the insulin kit was 0.5 $\mathrm{mg} / \mathrm{L}$.

\section{Muscle Strength Analysis}

To measure muscle strength, hand dynamometer (YAGAMI DM-100) was applied. The sum of maximum left and right hands strength were recorded 3 times as mean muscle strength.

\section{Statistical Analysis}

The data was analyzed using SPSS software, version 20.0 and the graphs and tables were edited applying 
Excel software. All data had normal distribution, as demonstrated by the Kolmogorov-Smirnov and Levene tests. The analysis of differences within and betweensubjects presented by analysis of covariance (ANCOVA) and post hoc Bonferroni tests, determined the significance of the differences between the groups. The significance was accepted at $P \leq 0.05$.

\section{Results}

Table 1 shows the demographic characteristics of the subjects in each experimental group.

Table 2 shows the clinical variables before and after intervention resistance protocols. A significant increase was observed in protein synthesis, serum insulin, as well as muscle strength in pre-test and post-test in all groups after the implementation of the resistant and supplemented protocols.

Figure 1 displays the urine nitrogen changes after 8-week intervention. The urine nitrogen level significantly increased in RT and RTLS groups $(P<0.05)$. Besides, in inter-group comparisons, there was a significant difference between RT and C groups, RT and RTLS groups, and also LS and RTLS groups $(P<0.05)$. There was no statistically significant difference between LS and $C$ groups.

Figure 2 demonstrates muscle mass changes after 8-week intervention. The muscle mass significantly increased in RT and RTLS groups $(P<0.05)$. In addition, intergroup comparisons revealed that there was a significant difference between RT and C groups, RT and RTLS groups, as well as LS and RTLS groups $(P<0.05)$. There was no statistically significant difference between LS and C groups.

Figure 3 shows serum insulin changes after 8-week intervention. The serum insulin was significantly different between RTLS and RT groups $(P<0.05)$. Furthermore, in inter-group comparisons, a significant difference was observed between the RT and $\mathrm{C}$ groups $(P<0.05)$, RTLS and LS groups $(P<0.05)$.

Figure 4 represents muscle strength changes after the 8 -week intervention. The muscle strength significantly increased in RTLS, RT, and LS groups $(P<0.05)$. In addition, in inter-group comparisons a significant difference was observed between S and C groups, RT and C groups, RT and RTLS groups, as well as LS and RTLS groups $(P<0.05)$.

\section{Discussion}

The results of this study indicated that 8-week resistance training and leucine supplementation significantly increased urine nitrogen levels and muscle mass. However, this increase in LS group is not significantly different from that of $\mathrm{C}$ group, which is probably related to exercise duration and the amount of supplementation. Resistance training, through activating mTOR, leads to an increase in 4E-BP1 and S6K1, and the binding of mRNA to the ribosome. Thus, the protein synthesis rate increases. ${ }^{20,21}$ Several studies have highlighted the role of resistance exercise in stimulation of protein synthesis in youth and elderly. Therefore, resistance exercises for 2-6 months (1 or 2 sessions per week) can significantly stimulate protein synthesis in the elderly. ${ }^{22}$ Yarasheski et al indicated that after 3 months of resistance training with the exercise intensity of 65\%-100\% 1RM, the muscle mass increased. Consequently, the rate protein synthesis increases in older women and men. ${ }^{23}$ Fry et al reported the effects of resistance training with the intensity of exercise $70 \% 1 \mathrm{RM}$ on protein synthesis in the elderly. They found a significant increase in protein synthesis. ${ }^{24}$ The results revealed that the use of leucine amino acid after resistance training stimulated skeletal muscle protein synthesis more than resistance training in the absence of supplementation. Kim et al showed that resistance training and the consumption of leucine supplement for 3 months have increased the muscle mass and protein synthesis. ${ }^{25}$ Similarly, Panton et al reported that the muscle mass increased after consuming 3 grams of leucine supplement during 4 weeks of resistance training in the elderly. ${ }^{26}$ The animal model researches indicated that leucine consumption and resistance training increases anabolic processes and inhibits catabolic pathways in skeletal muscle. In the Gil and Kim studies, after eight weeks of resistance training and leucine supplementation, mTOR increased the synthesis of muscle protein in mice. ${ }^{21}$

Another important finding in the present study was the increase in serum insulin levels after eight weeks in RTLS, RT, and LS groups. However, this increase did not significantly differ between LS and C groups; these findings are probably related to the duration and amount of supplementation for optimal and effective adaptation to increase serum insulin levels in the elderly men of LS group. Besides, there was a significant difference between

Table 1. Individual Characteristics of the Subjects

\begin{tabular}{|c|c|c|c|c|}
\hline \multirow{2}{*}{ Variable } & \multicolumn{4}{|c|}{ Groups } \\
\hline & $C(n=10)$ & RTLS $(n=10)$ & $\mathrm{RT}(\mathrm{n}=10)$ & LS $(n=10)$ \\
\hline Age (year) & $62.10 \pm 1.44$ & $60.90 \pm 2.02$ & $61.10 \pm 3.34$ & $62.20 \pm 2.44$ \\
\hline Height (cm) & $167.90 \pm 8.88$ & $165.10 \pm 8.07$ & $166.80 \pm 4.68$ & $172.50 \pm 7.80$ \\
\hline Weight (kg) & $59.05 \pm 3.62$ & $55.93 \pm 2.66$ & $59.94 \pm 3.80$ & $66.10 \pm 3.92$ \\
\hline $\mathrm{BMI}\left(\mathrm{kg} / \mathrm{m}^{2}\right)$ & $21.06 \pm 2.13$ & $20.62 \pm 1.95$ & $21.54 \pm 1.09$ & $22.26 \pm 1.49$ \\
\hline
\end{tabular}

The data or the values are means \pm standard deviation.

RTLS: Resistance Training and Leucine Supplement, RT: Resistance Training, LS: leucine Supplement, C: Control. 
Table 2. Research Variables Before and After Protocol

\begin{tabular}{|c|c|c|c|c|c|c|c|}
\hline \multirow{2}{*}{ Group } & \multirow{2}{*}{ Variable } & \multicolumn{2}{|c|}{ Stage } & \multicolumn{2}{|c|}{ Within Group } & \multicolumn{2}{|c|}{ Between Group } \\
\hline & & Pre-test & Post- test & $\mathbf{F}$ & $\boldsymbol{P}$ & $\mathbf{F}$ & $P$ \\
\hline \multirow{4}{*}{$\begin{array}{l}\text { Nitrogen urine } \\
(\mathrm{mg} / \mathrm{dL})\end{array}$} & RTLS & $1327.26 \pm 44.60$ & $1602.12 \pm 70.0$ & \multirow{4}{*}{33.65} & \multirow{4}{*}{$0.001^{*}$} & \multirow{4}{*}{38.86} & \multirow{4}{*}{$0.001 *$} \\
\hline & RT & $1346.16 \pm 38.60$ & $1408.47 \pm 56.83$ & & & & \\
\hline & LS & $1399.20 \pm 41.69$ & $2140.18 \pm 80.39$ & & & & \\
\hline & C & $1362.87 \pm 50.90$ & $1230.01 \pm 53.60$ & & & & \\
\hline \multirow{4}{*}{ Muscle mass (kg) } & RTLS & $40.36 \pm 0.71$ & $44.23 \pm 0.81$ & \multirow{4}{*}{127.50} & \multirow{4}{*}{$0.001^{*}$} & \multirow{4}{*}{10.96} & \multirow{4}{*}{$0.001 *$} \\
\hline & RT & $38.76 \pm 0.69$ & $44.98 \pm 0.86$ & & & & \\
\hline & LS & $39.80 \pm 0.69$ & $43.94 \pm 0.83$ & & & & \\
\hline & C & $45.64 \pm 0.69$ & $38.23 \pm 0.91$ & & & & \\
\hline \multirow{4}{*}{$\begin{array}{l}\text { Serum insulin } \\
(\mu \mathrm{u} / \mathrm{mL})\end{array}$} & RTLS & $2.49 \pm 0.17$ & $4.86 \pm 0.21$ & \multirow{4}{*}{134.54} & \multirow{4}{*}{$0.001^{*}$} & \multirow{4}{*}{9.43} & \multirow{4}{*}{$0.001 *$} \\
\hline & RT & $2.92 \pm 0.16$ & $4.14 \pm 0.21$ & & & & \\
\hline & LS & $3.16 \pm 0.16$ & $3.99 \pm 0.22$ & & & & \\
\hline & $\mathrm{C}$ & $3.48 \pm 0.18$ & $3.22 \pm 0.22$ & & & & \\
\hline \multirow{4}{*}{ Strength muscle $(\mathrm{kg})$} & RTLS & $14.76 \pm 0.97$ & $23.50 \pm 0.62$ & \multirow{4}{*}{25.70} & \multirow{4}{*}{$0.001^{*}$} & \multirow{4}{*}{33.83} & \multirow{4}{*}{$0.001 *$} \\
\hline & RT & $14.80 \pm 0.42$ & $19.15 \pm 0.46$ & & & & \\
\hline & LS & $15.20 \pm 0.42$ & $17.57 \pm 0.45$ & & & & \\
\hline & C & $15.33 \pm 0.86$ & $14.89 \pm 0.61$ & & & & \\
\hline
\end{tabular}

* Significant difference between the subjects at the level of $P<0.01$.

The data means \pm standard deviation.

RTLS: Resistance Training and Leucine Supplement, RT: Resistance Training, LS: leucine Supplement, C: Control.

the serum insulin levels in the RTLS and LS groups. In the current study, resistance training, probably through stimulating muscle mass, may increase glucose uptake in the body and improve insulin levels. Insulin activates protein synthesis via $\mathrm{PI} 3-\mathrm{k}$ and $\mathrm{PKb} / \mathrm{AKT}$ pathways. This includes mTOR phosphorylation, following the activation of 2 proteins of $4 \mathrm{E}-\mathrm{BP} 1$ and $\mathrm{S} 6 \mathrm{~K} 1$, which initiates translation of the mRNA. ${ }^{8}$ Casperson et al reported that serum insulin levels were unchanged after 2 weeks of consuming leucine supplement. ${ }^{27}$ While in the study conducted by Volpi et alit was reported that, following the consumption of leucine supplement, insulin levels increased, so that insulin, as an important regulator of muscle protein, influenced protein synthesis in the elderly. ${ }^{20}$ Similarly, Sebastiano et al found that the consumption of eight grams of leucine supplement would

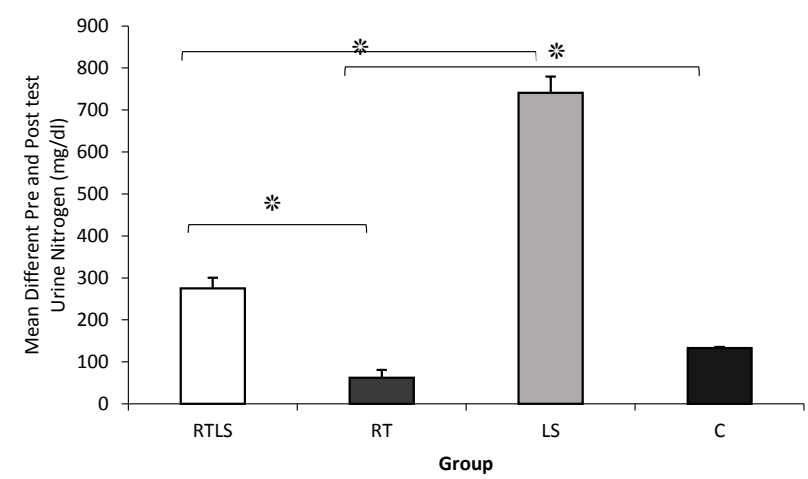

Figure 1. Urine Nitrogen Changes in Control Group $(C=10)$, Leucine Supplement Group (LS= 10), Resistance Training Group $(\mathrm{RT}=10)$, and Resistance Training and Leucine Supplement Group $(\mathrm{RTLS}=10)$.

* Significant difference between the groups at the level of $P<0.05$ increase serum insulin levels and insulin sensitivity in the elderly with sarcopenia. ${ }^{28}$

The results of the present study showed a significant increase in muscle strength eight weeks after resistance training and supplementation of leucine in elderly men with sarcopenia. However, it seems that the application of these 2 interventional factors (exercises and supplement) is accompanied by increased muscle strength, which indicates the interactive effect of these 2 methods. The results of this study showed that resistance training increased muscle strength by increasing muscle mass, but the exercise group demonstrated higher muscle strength when consuming supplements. The amino acid leucine as a regulator of protein metabolism increases the synthesis of skeletal muscle protein and reduces the rate of muscle breakdown and muscle relaxation. ${ }^{29}$ Therefore, the

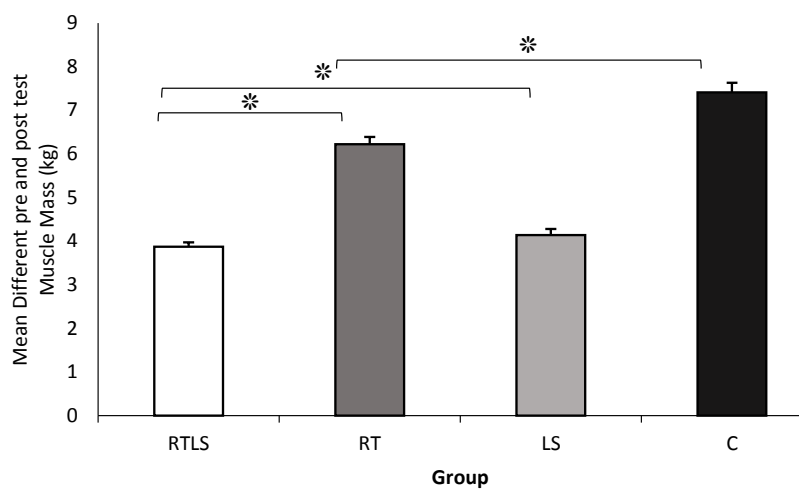

Figure 2. Muscle Mass Changes in Control Group $(C=10)$, Leucine Supplement Group (LS=10), Resistance Training Group (RT=10), and Resistance Training and Leucine Supplement Group (RTLS= $10)$.

* Significant difference between the groups at the level of $P<0.05$ 


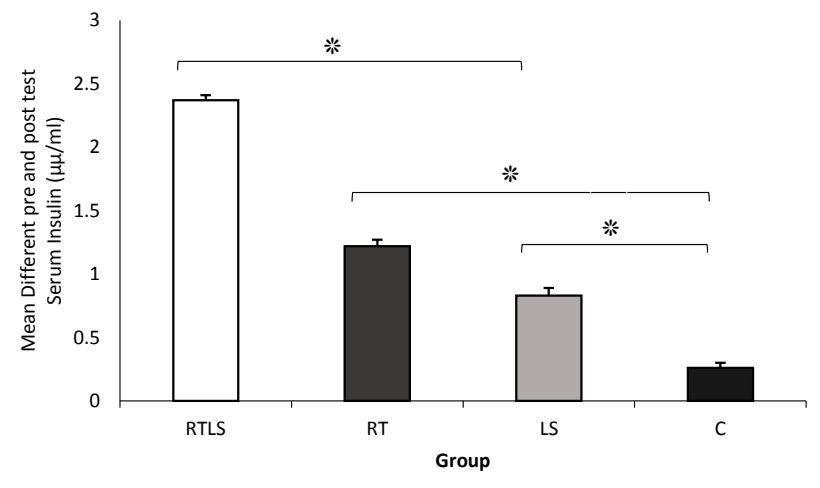

Figure 3. Serum Insulin Changes in Control Group $(C=10)$, Leucine Supplement Group (LS=10), Resistance Training Group (RT=10), and Resistance Training and Leucine Supplement Group (RTLS= 10).

* Significant difference between the groups at the level of $P<0.05$

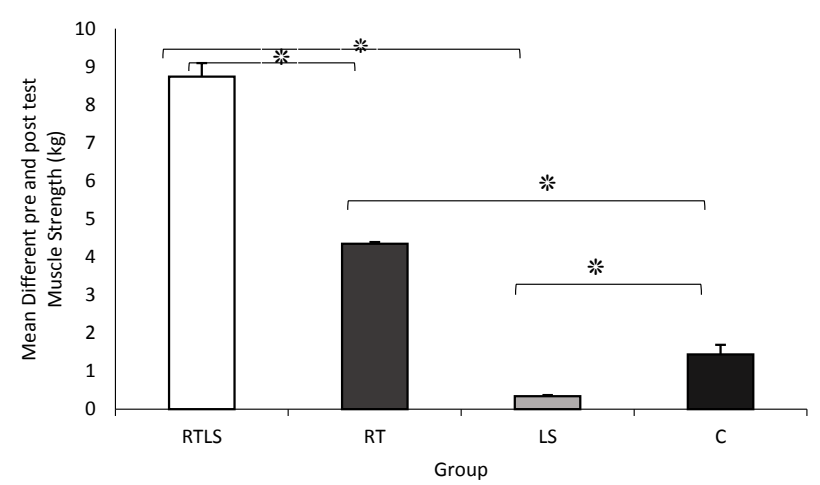

Figure 4. Muscle Strength Changes in Control Group $(C=10)$, Leucine Supplement Group ( $L S=10)$, Resistance Training Group $(\mathrm{RT}=10)$, and Resistance Training and Leucine Supplement Group $(\mathrm{RTLS}=10)$.

* Significant difference between the groups at the level of $P<0.05$

supplementation of leucine after physical activity resulted in increased muscle mass and skeletal muscle strength. Kim's study revealed that the use of leucine amino acids leads to increased muscle strength. However, when leucine supplementation with resistance training was consumed, increase in muscle strength and muscle mass was observed. ${ }^{15}$, A study by Meredith et al showed that after a period of resistance training and supplementation of leucine, muscle mass increased, while there was no changes in muscle strength..$^{30}$ In general, the investigations demonstrated that leucine and resistance training lead to improvements in muscle strength and muscle mass and, subsequently, reduce muscle atrophy and sarcopenia in the elderly. ${ }^{6}$

\section{Conclusion}

The results indicated that the muscle protein synthesis increased after resistance training in skeletal muscle. This implies that the rate of sarcopenia may be reduced as a result of resistance training. In addition, resistance training along with consuming leucine supplement was effective in improving muscle strength.

\section{Ethical Approval}

This research has been ethically approved by Kerman Medical Sciences University under the code of IR.Kmu. REC.1395.359.

\section{Competing Interests}

Authors declare that they have no competing interests.

\section{Acknowledgments}

We would like to express thanks to all the colleagues and subjects who engaged in this study.

\section{References}

1. Deutz NE, Bauer JM, Barazzoni R, et al. Protein intake and exercise for optimal muscle function with aging: recommendations from the ESPEN Expert Group. Clin Nutr. 2014;33(6):929-936. doi:10.1016/j.clnu.2014.04.007.

2. Curcio F, Ferro G, Basile C, et al. Biomarkers in sarcopenia: a multifactorial approach. Exp Gerontol. 2016;85:1-8. doi:10.1016/j.exger.2016.09.007.

3. Manders RJ, Little JP, Forbes SC, Candow DG. Insulinotropic and muscle protein synthetic effects of branched-chain amino acids: potential therapy for type 2 diabetes and sarcopenia. Nutrients. 2012;4(11):1664-1678. doi:10.3390/nu4111664.

4. Tramontano A, Veronese N, Sergi G, et al. Prevalence of sarcopenia and associated factors in the healthy older adults of the Peruvian Andes. Arch Gerontol Geriatr. 2017;68:4954. doi:10.1016/j.archger.2016.09.002.

5. Balagopal P, Schimke JC, Ades P, Adey D, Nair KS. Age effect on transcript levels and synthesis rate of muscle MHC and response to resistance exercise. Am J Physiol Endocrinol Metab. 2001;280(2):E203-E208. doi:10.1152/ ajpendo.2001.280.2.E203.

6. Troulinaki K, Tavernarakis N. Protein Synthesis and Ageing.

7. Kim J-S, Wilson JM, Lee S-R. Dietary implications on mechanisms of sarcopenia: roles of protein, amino acids and antioxidants. J Nutr Biochem. 2010;21(1):1-13. doi:10.1016/j.jnutbio.2009.06.014.

8. Morley JE, Argiles JM, Evans WJ, et al. Nutritional recommendations for the management of sarcopenia. J Am Med Dir Assoc. 2010;11(6):391-396. doi:10.1016/j. jamda.2010.04.014.

9. Wang X, Proud CG. The mTOR pathway in the control of protein synthesis. Physiology. 2006;21(5):362-369. doi:10.1152/physiol.00024.2006.

10. Norton LE, Layman DK. Leucine regulates translation initiation of protein synthesis in skeletal muscle after exercise. J Nutr. 2006;136(2):533S-537S. doi:10.1093/ jn/136.2.533S.

11. Kimball SR, Farrell PA, Jefferson LS. Invited Review: Role of insulin in translational control of protein synthesis in skeletal muscle by amino acids or exercise. J Appl Physiol. 2002;93(3):1168-1180. doi:10.1152/ japplphysiol.00221.2002. 
12. Yu J. The etiology and exercise implications of sarcopenia in the elderly. Int J Nurs Sci. 2015;2(2):199-203. doi:10.1016/j. ijnss.2015.04.010.

13. Kumar V, Selby A, Rankin D, et al. Age-related differences in the dose-response relationship of muscle protein synthesis to resistance exercise in young and old men. J Physiol. 2009;587(1):211-217. doi:10.1113/jphysiol.2008.164483.

14. Dickinson JM, Volpi E, Rasmussen BB. Exercise and nutrition to target protein synthesis impairments in aging skeletal muscle. Exerc Sport Sci Rev. 2013;41(4):216. 10.1097/JES.0b013e3182a4e699.

15. Drummond MJ, Dreyer HC, Pennings B, et al. Skeletal muscle protein anabolic response to resistance exercise and essential amino acids is delayed with aging. J Appl Physiol. 2008;104(5):1452-1461. doi:10.1152/ japplphysiol.00021.2008.

16. Waters D, Baumgartner R, Garry P, Vellas B. Advantages of dietary, exercise-related, and therapeutic interventions to prevent and treat sarcopenia in adult patients: an update. Clin Interv Aging. 2010;5:259.

17. Willoughby D, Stout J, Wilborn C. Effects of resistance training and protein plus amino acid supplementation on muscle anabolism, mass, and strength. Amino Acids. 2007;32(4):467-477. doi 10.1007/s00726-006-0398-7.

18. Hashemi R, Shafiee G, Motlagh AD, et al. Sarcopenia and its associated factors in Iranian older individuals: results of SARIR study. Arch Gerontol Geriatr. 2016;66:18-22. doi:10.1016/j.archger.2016.04.016.

19. Hong J, Kim J, Kim SW, Kong H-J. Effects of home-based tele-exercise on sarcopenia among community-dwelling elderly adults: body composition and functional fitness. Exp Gerontol. 2017;87:33-39. doi:10.1016/j.exger.2016.11.002.

20. Volpi E, Kobayashi H, Sheffield-Moore M, Mittendorfer B, Wolfe RR. Essential amino acids are primarily responsible for the amino acid stimulation of muscle protein anabolism in healthy elderly adults. Am J Clin Nutr. 2003;78(2):250258. doi:10.1093/ajcn/78.2.250.

21. Gil JH, Kim CK. Effects of different doses of leucine ingestion following eight weeks of resistance exercise on protein synthesis and hypertrophy of skeletal muscle in rats. J Exerc Nutrition Biochem. 2015;19(1):31. doi: 10.5717/jenb.2015.19.1.31.

22. Cooper LA, Brown SL, Hocking E, Mullen AC. The role of exercise, milk, dairy foods and constituent proteins on the prevention and management of sarcopenia. International Journal of Dairy Technology. 2016;69(1):1321. doi:10.1111/1471-0307.12280.

23. Yarasheski KE, Pak-Loduca J, Hasten DL, Obert KA, Brown MB, Sinacore DR. Resistance exercise training increases mixed muscle protein synthesis rate in frail women and men $\geq 76$ yr old. Am J Physiol Endocrinol Metab. 1999;277(1):E118-E125. doi:10.1152/ ajpendo.1999.277.1.E118.

24. Fry CS, Drummond MJ, Glynn EL, et al. Skeletal muscle autophagy and protein breakdown following resistance exercise are similar in younger and older adults. Journals of Gerontology Series A: Biomedical Sciences and Medical Sciences. 2012;68(5):599-607. doi:10.1093/gerona/gls209.

25. Kim HK, Suzuki T, Saito K, et al. Effects of exercise and amino acid supplementation on body composition and physical function in community-dwelling elderly Japanese sarcopenic women: a randomized controlled trial. J Am Geriatr Soc. 2012;60(1):16-23. doi:10.1111/j.15325415.2011.03776.x.

26. Panton LB, Rathmacher JA, Baier S, Nissen S. Nutritional supplementation of the leucine metabolite $\beta$-hydroxy$\beta$-methylbutyrate (hmb) during resistance training1. Nutrition. 2000;16(9):734-739. doi:10.1016/S08999007(00)00376-2.

27. Casperson SL, Sheffield-Moore M, Hewlings SJ, PaddonJones D. Leucine supplementation chronically improves muscle protein synthesis in older adults consuming the RDA for protein. Clin Nutr. 2012;31(4):512-519. doi:10.1016/j.clnu.2012.01.005.

28. Solerte SB, Gazzaruso C, Bonacasa R, et al. Nutritional supplements with oral amino acid mixtures increases wholebody lean mass and insulin sensitivity in elderly subjects with sarcopenia. Am J Cardiol. 2008;101(11):S69-S77. doi:10.1016/j.amjcard.2008.03.004.

29. van Schenau GJI, de Koning JJ, de Groot G. Optimisation of sprinting performance in running, cycling and speed skating. Sports Med. 1994;17(4):259-275.

30. Meredith CN, Frontera WR, O’Reilly KP, Evans WJ. Body composition in elderly men: effect of dietary modification during strength training. J Am Geriatr Soc. 1992;40(2):155162. doi:10.1111/j.1532-5415.1992.tb01937.x. 